\title{
Choice-Consistent Resolutions of the Efficiency-Equity Trade-Off
}

\author{
Koichi Tadenuma \\ Faculty of Economics, Hitotsubashi University. Kunitachi, Tokyo 186-8601, \\ Japan. E-mail: tadenuma@econ.hit-u.ac.jp
}

Summary. In a standard framework of choice theory, we formulate two contrasting principles for social choice under the efficiency-equity trade-off. The equity-first principle states that we should select from equitable allocations if any, but if the equity criterion is not at all effective for selection either because all the available allocations are equitable or because no allocation is equitable, we should select from Pareto efficient allocations. The efficiency-first principle switches the roles of the equity criterion and the efficiency criterion above. We examine the choice-consistency properties, known as Path Independence (Arrow, 1963) and Contraction Consistency (Chernoff, 1954), of the social choice correspondences satisfying the equity-first or the efficiency-first principle. Several possibility and impossibility theorems are obtained, which indicate that possibility of consistent social decisions depends crucially on which principle we take as well as what is the precise notion of equity.

Key words: equity, efficiency, lexicographic composition, choice-consistency, path-independence

\section{Introduction}

It is not rare that multiple criteria are applied to make individual or social decisions. In the context of resource allocation problems, most prominent criteria are efficiency and equity of allocations. Pareto efficiency is probably the most widely accepted criterion among economists, but it is silent about the distributional equity of allocations. On the other hand, several concepts of equity have been proposed and extensively studied in welfare economics. Two of them are central: noenvy (Foley, 1967, and Kolm, 1972) and egalitarian-equivalence (Pazner and Schmeidler, 1978). We say that an allocation is envy-free if no agent 
prefers the consumption bundle of any other agent, and that an allocation is egalitarian-equivalent if there is a consumption bundle, called the reference bundle, such that every agent is indifferent between the bundle and his own.

However, as Kolm (1972) and Feldman and Kirman (1974) pointed out, there is a fundamental conflict between the Pareto efficiency criterion and the no-envy criterion. There often exist two allocations $x$ and $y$ such that $x$ is Pareto superior to $y$ whereas $x$ is not envy-free but $y$ is. If these two allocations $\{x, y\}$ are the only policy options available at the time, we cannot attain an allocation that is both Pareto efficient in $\{x, y\}$ and envy-free, but we have to choose either the efficient allocation or the envy-free allocation. The same kind of conflict also arises between Pareto efficiency and egalitarian-equivalence, as shown by Tadenuma (2005).

If two criteria of decision-making are incompatible simultaneously, we have to give priority to one criterion. That is, we take one criterion as the first and the other as the second, and apply them in the lexicographic order. This paper formalizes the idea in a standard framework of social choice correspondences in economic environments. Given the first and the second criterion, we require that choice should always be made from the allocations satisfying the first criterion whenever there are any. The second criterion should then be applied when the first criterion is not at all effective as a guide for selection, namely, either when all the available allocations satisfy the first criterion or when there is no such allocation at all. A stronger version of this condition may be obtained by requiring that all the allocations satisfying the second criterion should be selected in the latter case.

Besides the socially desirable properties of selected allocations, another important requirement for social choice correspondences is choiceconsistency. Especially, Path Independence is crucial. It implies "the independence of the final choice from the path to it" (Arrow, 1963, p. 120). Path independence is an indispensable property of social choice rules. Were it violated, some arbitrary agenda controls could affect the final choice, which is clearly undesirable.

Another natural choice-consistency condition, which is weaker than Path Independence, is Contraction Consistency. This says that if an allocation is chosen from a set $S$ of available allocations, then it should also be chosen from any subset $T$ of $S$ as long as it is still available.

The purpose of this paper is to examine possibility of consistent choices under the efficiency-first and equity-second principle or the equity-first and efficiency-second principle. We show several impossi- 
bility theorems on the existence of social choice correspondences satisfying the efficiency-first and equity-second principle with the concepts of no-envy and egalitarian-equivalence, and Contraction Consistency. However, if we restrict the range of reference bundles for egalitarianequivalence to a fixed ray from the origin, then there exists a social choice correspondence satisfying the efficiency-first and equity-secondas-egalitarian-equivalence principle and Path Independence. But even for this case, the stronger versions of properties representing the principle is incompatible with Path Independence.

Turning to the equity-first and efficiency-second principle, we also obtain impossibility and possibility results on the existence of social choice correspondences satisfying the principle and choice-consistency properties. It turns out that the borderline between possibility and impossibility is quite subtle. If our equity criterion selects only allocations with no-envy at all, then there exists a social choice correspondence satisfying the equity-first principle and Path Independence. Moreover, we obtain a characterization of the social choice correspondence by using the stronger versions of properties representing the principle and Path Independence. However, if we select allocations with "minimal-envy" according to the measure of envy-instances introduced by Suzumura (1996), which is based on the set-inclusions of envy relations, then no social choice correspondence satisfies the equity-first principle and Contraction Consistency together. In contrast, if the equity criterion selects allocations with "least-envy" in the sense that the number of envy-instances is the smallest (Feldman and Kirman, 1974), then compatibility with Path Independence is retained.

The rest of this paper is organized as follows. Section 2 defines basic concepts and notation, and Section 3 introduces various properties of social choice correspondences that represent the efficiency first principle or the equity first principle, and choice-consistency. In Section 4, we review the fundamental conflict between efficiency and equity. Section 5 examines choice-consistency of the social choice correspondences satisfying the efficiency first principle, and Section 6 turns to the equity first principle. In Section 7, we study the equity first principle with the notion of minimal-envy. Section 8 contains concluding remarks.

\section{Basic Definitions and Notation}

The framework and several fundamental results are due to Tadenuma (1998). There are $n$ agents and $m$ infinitely divisible goods, where $n$ and $m$ are some integers with $n, m \geq 2$. Let $N=\{1, \cdots, n\}$ be the 
set of agents. Denoting by $\mathbb{R}$ the set of real numbers, the set $\mathbb{R}_{+}^{m}$ is the consumption set of each agent. Let $\mathcal{R}$ be the class of preference relations on $\mathbb{R}_{+}^{m}$ that are reflexive, transitive, complete, continuous, and monotonic. Each agent $i \in N$ is endowed with a preference relation $R_{i} \in \mathcal{R}$. The strict preference relation and the indifference relation of agent $i$ are denoted by $P_{i}$ and $I_{i}$, respectively. A list of preference relations, $\left(R_{i}\right)_{i \in N} \in \mathcal{R}^{n}$, is called a preference profile, and denoted by $R_{N}$.

An allocation is a vector $x=\left(x_{1}, \cdots, x_{n}\right) \in \mathbb{R}_{+}^{m n}$ where each $x_{i}=$ $\left(x_{i 1}, \cdots, x_{i m}\right) \in \mathbb{R}_{+}^{m}$ is a consumption bundle of agent $i \in N$. The set of all allocations is denoted by $X$. We set $X=\mathbb{R}_{+}^{m n}$ for simplicity of presentation. However, we might impose a resource constraint on $X$. For example, given a total amount of resources $\Omega \in \mathbb{R}_{+}^{m}$, define the set of all feasible allocations with no free disposal as $X:=\{x \in$ $\left.\mathbb{R}_{+}^{m n} \mid \sum_{i=1}^{n} x_{i}=\Omega\right\}$. All the results in this paper hold on this more restricted set of allocations. Let $\mathcal{S}$ be the set of all non-empty finite subsets of $X$.

Let a preference profile $R_{N} \in \mathcal{R}^{n}$ be given. An allocation $x \in X$ is weakly Pareto superior to an allocation $y \in X$ for $R_{N}$ if $x_{i} R_{i} y_{i}$ for all $i \in N$. We write $x \succsim_{P\left(R_{N}\right)} y$ if $x$ is weakly Pareto superior to $y$. Let $\succ_{P\left(R_{N}\right)}$ be the strict part of $\succsim_{P\left(R_{N}\right)} \cdot{ }^{1}$ An allocation $x \in X$ is Pareto superior to an allocation $y \in X$ for $R_{N}$ if $x \succ_{P\left(R_{N}\right)} y$. For each $S \in \mathcal{S}$, an allocation $x \in S$ is Pareto efficient in $S$ for $R_{N}$ if there is no allocation $y \in S$ such that $y \succ_{P\left(R_{N}\right)} x$. Let $P\left(R_{N}, S\right)$ be the set of Pareto efficient allocations in $S$ for $R_{N}$.

An allocation $x \in X$ is envy-free for $R_{N}$ if $x_{i} R_{i} x_{j}$ for all $i, j \in N$. For each $S \in \mathcal{S}$, let $F\left(R_{N}, S\right)$ be the set of envy-free allocations in $S$ for $R_{N}$. An allocation $x \in X$ is egalitarian-equivalent for $R_{N}$ if there is a consumption bundle $x_{0} \in \mathbb{R}_{+}^{m}$ such that for all $i \in N, x_{i} I_{i} x_{0}$. Then, the bundle $x_{0}$ is called a reference bundle for $x$. For each $S \in \mathcal{S}$, let $E\left(R_{N}, S\right)$ be the set of egalitarian-equivalent allocations in $S$ for $R_{N}$. Particular subclasses of egalitarian-equivalent allocations are often studied in the literature. Let $\bar{r} \in \mathbb{R}_{++}^{m}$ be a given vector. An allocation $x \in X$ is egalitarian-equivalent for a fixed reference ray with $\bar{r}$ for $R_{N}$ or simply $\bar{r}$-egalitarian-equivalent for $R_{N}$ if there is a real number $t \in \mathbb{R}$ such that for all $i \in N, x_{i} I_{i} t \bar{r}$. For each $S \in \mathcal{S}$, let $E_{\bar{r}}\left(R_{N}, S\right)$ be the set of $\bar{r}$-egalitarian-equivalent allocations in $S$ for $R_{N}$.

A social choice correspondence is a set-valued function $\varphi: \mathcal{R}^{n} \times \mathcal{S} \rightarrow$ $\mathcal{S}$ such that $\varphi\left(R_{N}, S\right) \subseteq S$ for all $\left(R_{N}, S\right) \in \mathcal{R}^{n} \times \mathcal{S}$. A social choice

\footnotetext{
${ }^{1}$ Given a binary relation $\succsim$, its strict part $\succ$ is defined as $x \succ y \Leftrightarrow x \succsim y$ and $y \succsim x$.
} 
correspondence is interpreted as follows. Each $S \in \mathcal{S}$ is interpreted as the set of allocations available, which may be termed an environment following (Arrow, 1963, p. 15). Then $\varphi\left(R_{N}, S\right)$ is the set of socially desirable allocations in the given environment $S$ when the preferences of the agents are $R_{N}$. A fundamental example of a social choice correspondence is the Pareto correspondence, denoted by $P$, which associates with each $\left(R_{N}, S\right) \in \mathcal{R}^{n} \times \mathcal{S}$ the set of all Pareto efficient allocations in $S$ for $R_{N}$. The No-Envy correspondence, the Egalitarian-Equivalence correspondence, and the $\bar{r}$-Egalitarian-Equivalence correspondence, denoted by $F, E$, and $E_{\bar{r}}$, respectively, can be defined analogously.

A remark should be in order on the domain of social choice correspondences. The domain consists of the preferences domain $\mathcal{R}$ and the alternatives domain $\mathcal{S}$. As in many contributions in the literature of social choice theory, we assume that $\mathcal{S}$ is the class of all finite subsets of $X$. Our major interest here is not in investigating what are "optimal" allocations in the set of all technologically feasible allocations. There are many situations in which only a finite number of policy options are at issue at any one time. In such situations, we are rather interested in examining "consistency" of social choices at different times, or under expansions, contractions, or partitions of alternatives available at hand. To that end, our choice of $\mathcal{S}$ would be appropriate.

\section{The Axioms}

This section introduces a variety of desirable properties of social choice correspondences, which we call "axioms". In the rest of the paper, we denote by $\varphi$ a social choice correspondence.

The first axiom is familiar. It means that we should always select from Pareto efficient allocations whenever they exist. ${ }^{2}$

Pareto Efficiency. For all $\left(R_{N}, S\right) \in \mathcal{R}^{n} \times \mathcal{S}$, if $P\left(R_{N}, S\right) \neq \emptyset$, then $\varphi\left(R_{N}, S\right) \subseteq P\left(R_{N}, S\right)$.

The next three axioms require that only equitable allocations should be chosen whenever there are any.

No-Envy. For all $\left(R_{N}, S\right) \in \mathcal{R}^{n} \times \mathcal{S}$, if $F\left(R_{N}, S\right) \neq \emptyset$, then $\varphi\left(R_{N}, S\right) \subseteq$ $F\left(R_{N}, S\right)$.

\footnotetext{
${ }^{2}$ Note that, in our present model, there always exists a Pareto efficient allocation in $S \in \mathcal{S}$ since $S$ is finite. To keep symmetry in the definitions of axioms concerning efficiency and equity, and to present a definition which may be applicable to other contexts where there may not exist Pareto efficient allocations, we include the condition that $P\left(R_{N}, S\right) \neq \emptyset$ in the definition of this axiom.
} 
Egalitarian-Equivalence: For all $\left(R_{N}, S\right) \in \mathcal{R}^{n} \times \mathcal{S}$, if $E\left(R_{N}, S\right) \neq \emptyset$, then $\varphi\left(R_{N}, S\right) \subseteq E\left(R_{N}, S\right)$.

$\bar{r}$-Egalitarian-Equivalence: For all $\left(R_{N}, S\right) \in \mathcal{R}^{n} \times \mathcal{S}$, if $E_{\bar{r}}\left(R_{N}, S\right) \neq$ $\emptyset$, then $\varphi\left(R_{N}, S\right) \subseteq E_{\bar{r}}\left(R_{N}, S\right)$.

Even if the efficiency criterion is taken as the first principle for social choice, equity criteria should be used when the efficiency criterion is not at all effective as a guide for selection: either when all the available allocations are efficient or when no available allocation is so.

P-Conditional No-Envy ${ }^{3}$ : For all $\left(R_{N}, S\right) \in \mathcal{R}^{n} \times \mathcal{S}$, if (i) $P\left(R_{N}, S\right)=S$ or $P\left(R_{N}, S\right)=\emptyset$, and (ii) $F\left(R_{N}, S\right) \neq \emptyset$, then $\varphi\left(R_{N}, S\right) \subseteq F\left(R_{N}, S\right)$.

The next axiom strengthens P-Conditional No-Envy. It means that if either all the available allocations are efficient or no available allocation is efficient, then all the envy-free allocations should be recommended. In other words, it claims that we should not discriminate between allocations that equally satisfy the efficiency and equity criteria defined explicitly as axioms. ${ }^{4}$

P-Conditional No-Envy Inclusion: For all $\left(R_{N}, S\right) \in \mathcal{R}^{n} \times \mathcal{S}$, if (i) $P\left(R_{N}, S\right)=S$ or $P\left(R_{N}, S\right)=\emptyset$, and (ii) $F\left(R_{N}, S\right) \neq \emptyset$, then $\varphi\left(R_{N}, S\right)=F\left(R_{N}, S\right)$.

By simply replacing the correspondence $F$ with each of the correspondences $E$ and $E_{\bar{r}}$ in the above definitions, we define PConditional Egalitarian-Equivalence and P-Conditional $\bar{r}$ Egalitarian-Equivalence, respectively, and their corresponding stronger versions.

Turnig now to the equity-first and efficiency-second principle, we define the counterparts of the above axioms. Let a social choice correspondence $\Psi \in\left\{F, E, E_{\bar{r}}\right\}$ be given. (The correspondence $\Psi$ is one of

\footnotetext{
${ }^{3}$ The capital letter $\mathbf{P}$ stands for the Pareto correspondence.

4 The issue of whether we should take the stronger version of conditional equity or efficiency may be related with the issue of full versus partial implementation of social choice correspondences. Thomson (1996) made an argument in support of full implementation as follows: "one should make sure that the complete list of desired properties of correspondences have been identified, and then identify the class of correspondences satisfying them. If all the properties are satisfied only by $F$ (that is, if $F$ is characterized by these properties), then full implementation of $F$ is indeed what we should be after." (p. 135)
} 
the three "equity correspondences".) If the equity criterion described by $\Psi$ is accepted as the first selection principle, we may still apply the efficiency criterion when all the allocations available are equitable, or when there is no equitable allocation at all.

$\Psi$-Conditional Pareto Efficiency: For all $\left(R_{N}, S\right) \in \mathcal{R}^{n} \times \mathcal{S}$, if (i) $\Psi\left(R_{N}, S\right)=S$ or $\Psi\left(R_{N}, S\right)=\emptyset$, and (ii) $P\left(R_{N}, S\right) \neq \emptyset$, then $\varphi\left(R_{N}, S\right) \subseteq P\left(R_{N}, S\right)$.

As an example, when $\Psi=F$, we write the above axiom $\mathbf{F}$-Conditional Pareto Efficiency. ${ }^{5}$ A strengthening of $\Psi$-Conditional Pareto Efficiency is the following.

$\Psi$-Conditional Pareto Inclusion: For all $\left(R_{N}, S\right) \in \mathcal{R}^{n} \times \mathcal{S}$, if (i) $\Psi\left(R_{N}, S\right)=S$ or $\Psi\left(R_{N}, S\right)=\emptyset$, and (ii) $P\left(R_{N}, S\right) \neq \emptyset$, then $\varphi\left(R_{N}, S\right)=P\left(R_{N}, S\right)$.

Next, we introduce several choice-consistency axioms. The first one is called Path Independence, which is due to Arrow (1963) and Plott (1973), and may be described as follows. Let $S$ be the set of available allocations, and $\left\{S_{1}, S_{2}\right\}$ be a partition of $S$. Suppose that we first choose desirable allocations $\varphi\left(S_{i}\right)$ from each $S_{i}(i=1,2)$, and next make the final choice from $\varphi\left(S_{1}\right) \cup \varphi\left(S_{2}\right)$, that is, from the "winners" of the first round. Then, Path Independence requires that for all partitions of $S$, the final choice should be the same, and hence the choice be independent of the way how to partition $S$. Therefore, path independent social choice rules are immune to any agenda control.

Path Independence: For all $R_{N} \in \mathcal{R}^{n}$, and all $S_{1}, S_{2} \in \mathcal{S}, \varphi\left(R_{N}, S_{1} \cup\right.$ $\left.S_{2}\right)=\varphi\left(R_{N}, \varphi\left(R_{N}, S_{1}\right) \cup \varphi\left(R_{N}, S_{2}\right)\right)$.

Path Independence implies the following choice-consistency condition, which was introduced by Chernoff (1954). Its intuitive meaning is also clear: Suppose that an allocation $x$ is chosen from a set $S_{1}$, and then the set of available alternatives is contracted to $S_{2} \subset S_{1}$, but the allocation $x$ is still available. Then, this allocation should be selected from the set $S_{2}$ as well.

Contraction Consistency: For all $R_{N} \in \mathcal{R}^{n}$ and all $S_{1}, S_{2} \in \mathcal{S}$ with $S_{2} \subseteq S_{1}, S_{2} \cap \varphi\left(S_{1}\right) \subseteq \varphi\left(S_{2}\right)$.

${ }^{5}$ The capital letter $\mathrm{F}$ stands for Freedom from envy. 
Ever since Arrow (1951), it has been a central issue in social choice theory whether social choice correspondences are rationalizable, that is, the choice described by the social choice correspondence from each set of available alternatives could be obtained by maximization of some "well-behaved" social preference relation. The question itself is of much theoretical interest, and moreover it is worth examining because various rationalizability conditions are logically related to choice-consistency conditions.

Let $\succ$ be an irreflexive and asymmetric binary relation on $X$, the interpretation of which is a strict social preference relation. ${ }^{6}$ For each $S \in \mathcal{S}$, denote by $M_{\succ}(S)$ the set of maximal elements of $\succ$ in $S$, that is,

$$
M_{\succ}(S):=\{x \in S \mid \text { There exists no } y \in S \text { such that } y \succ x\}
$$

Quasi-Transitive Rationalizability: ${ }^{7}$ For every $R_{N} \in \mathcal{R}^{n}$, there exists an irreflexive, asymmetric, and transitive binary relation $\succ_{\left(R_{N}\right)}$ on $X$ such that for all $S \in \mathcal{S}, \varphi\left(R_{N}, S\right)=M_{\succ_{\left(R_{N}\right)}}(S)$.

We say that a binary relation $\succ$ has a cycle if there exist a positive integer $K$ and $K$ allocations $x^{1}, \cdots, x^{K}$ such that $x^{k} \succ x^{k+1}$ for all $k$ with $1 \leq k \leq K-1$, and $x^{K} \succ x^{1}$. The binary relation $\succ$ is acyclic if it has no cycle. ${ }^{8}$

Acyclic Rationalizability: For every $R_{N} \in \mathcal{R}^{n}$, there exists an acyclic binary relation $\succ_{\left(R_{N}\right)}$ on $X$ such that for all $S \in \mathcal{S}, \varphi\left(R_{N}, S\right)=$ $M_{\succ_{\left(R_{N}\right)}}(S)$.

The conditions introduced above have the following logical relations. ${ }^{9}$ Quasi-Transitive Rationalizability implies both Acyclic Rationalizability and Path Independence, and each of the two conditions, Acyclic Rationalizability and Path Independence, implies Contraction Consistency. The converse of each statement does not hold true. Hence,

\footnotetext{
${ }^{6}$ It will be convenient for us to present the results by strict social preference relations $\succ$. However, we could alternatively use the reflexive and complete social preference relations $\succsim$ induced from $\succ$ as follows: For all $x, y \in X, x \succsim y$ if and only if $y \succ x$ does not hold.

7 The term "quasi-transitivity" is due to Sen (1970), which means transitivity of strict social preference relations.

${ }^{8}$ Note that if $\succ$ is acyclic, then by definition, it is irreflexive and asymmetric.

${ }^{9}$ See Suzumura (1983, chap. 3).
} 
Contraction Consistency may be considered as the minimal requirement of choice-consistency of social choice correspondences. It is also a necessary condition for any kind of rationalizability by a single binary relation, but it is not a sufficient condition even for Acyclic Rationalizability. ${ }^{10}$

Figure 1 summarizes the logical relations between the axioms. Each arrow indicates the direction of logical implication.

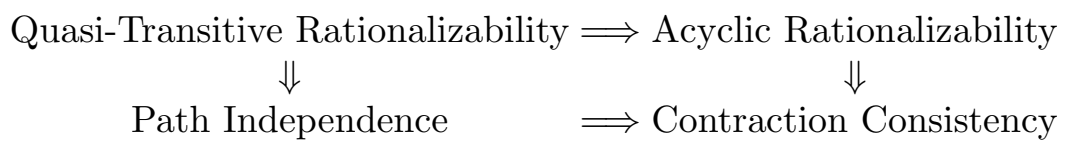

Figure 1: Logical relations of choice-consistency and rationalizability conditions

Our final axiom is an obvious requirement: Social choice rules should be able to select some allocations for any environment.

Non-Emptiness: For all $\left(R_{N}, S\right) \in \mathcal{R}^{n} \times \mathcal{S}, \varphi\left(R_{N}, S\right) \neq \emptyset$.

\section{Conflicts between Efficiency and Equity}

This section reviews the fundamental conflict between the Pareto efficiency criterion and the equity criteria. First, we observe the conflict between efficiency and no-envy, which was first pointed out by Kolm (1972).

Example 1 There are two agents $N=\{1,2\}$ and two goods $\{1,2\}$. The preferences of the agents are represented by the utility functions

$$
\begin{aligned}
& u_{1}\left(x_{11}, x_{12}\right)=x_{11} x_{12} \\
& u_{2}\left(x_{21}, x_{22}\right)=2 x_{21}+x_{22}
\end{aligned}
$$

\footnotetext{
$\overline{10}$ As a counter-example for the last claim, consider the social choice correspondence defined as follows: Choose $x^{0} \in X$. Define the correspondence $C$ by

$$
C(S)=S \text { otherwise. }
$$$$
C(S)=\left\{x^{0}\right\} \text { if } x^{0} \in S \text { and }|S| \geq 3
$$

It can be checked that the correspondence $C$ satisfies Contraction Consistency, but cannot be rationalized by any social preference relation. 
Let $x=\left(x_{1}, x_{2}\right)=\left(\left(1, \frac{46}{5}\right),\left(9, \frac{4}{5}\right)\right)$ and $y=\left(y_{1}, y_{2}\right)=\left(\left(\frac{11}{5}, \frac{22}{5}\right),\left(\frac{39}{5}, \frac{28}{5}\right)\right.$. Then, since $u_{1}\left(y_{1}\right)>u_{1}\left(x_{1}\right)$ and $u_{2}\left(y_{2}\right)>u_{2}\left(x_{2}\right)$, the allocation $y$ is Pareto superior to the allocation $x$. However, $x$ is envy-free because $u_{1}\left(x_{1}\right)>u_{1}\left(x_{2}\right)$ and $u_{2}\left(x_{2}\right)>u_{2}\left(x_{1}\right)$, whereas $y$ is not since $u_{1}\left(y_{2}\right)>$ $u_{1}\left(y_{1}\right)$. Now let $S=\{x, y\}$. Then, both the sets $P\left(R_{N}, S\right)$ and $F\left(R_{N}, S\right)$ are non-empty, but the intersection of the two sets is empty.

This example can be extended to the case of any finite numbers of agents and goods. Hence, we have the following impossibility.

Theorem 1. [Kolm (1972), Feldman and Kirman (1974), Suzumura (1981a)] There exists no social choice correspondence that satisfies Non-Emptiness, Pareto Efficiency, and No-Envy.

Next we show that the same kind of conflict may also arise between the Pareto efficiency criterion and the equity-as-egalitarian-equivalence criterion.

Example 2 There are two agents $N=\{1,2\}$ and two goods 1,2. The preferences of the agents are represented by the utility functions ${ }^{11}$

$$
\begin{aligned}
& u_{1}\left(x_{11}, x_{12}\right)=\min \left\{x_{11}, x_{12}\right\} \\
& u_{2}\left(x_{21}, x_{22}\right)=x_{21}+3 x_{22}
\end{aligned}
$$

Let $x=\left(x_{1}, x_{2}\right)=((3,11),(9,1))$ and $y=\left(y_{1}, y_{2}\right)=((8,8),(4,4))$. Let $\bar{r}=(1,1)$. Then, the allocation $y$ is Pareto superior to the allocation $x$. On the other hand, since $u_{1}\left(x_{1}\right)=3=u_{1}(3 \bar{r})$ and $u_{2}\left(x_{2}\right)=12=u_{2}(3 \bar{r})$, the allocation $x$ is $\bar{r}$-egalitarian-equivalent with $3 \bar{r}$ being the reference bundle. However, $y$ is not egalitarian-equivalent because for any bundle $z_{0}$ such that $u_{1}\left(z_{0}\right)=u_{1}\left(y_{1}\right), z_{0} \geq(8,8)$ and hence $u_{2}\left(z_{0}\right) \geq u_{2}(8,8)>u_{2}(4,4)=u_{2}\left(y_{2}\right)$. Let $S=\{x, y\}$. Then, both the sets $P\left(R_{N}, S\right)$ and $E\left(R_{N}, S\right)=E_{\bar{r}}\left(R_{N}, S\right)$ are non-empty, but the intersection of the two sets is empty.

Theorem 2. [Tadenuma (2005)] (i) There exists no social choice correspondence that satisfies Non-Emptiness, Pareto Efficiency, and Egalitarian-Equivalence. (ii) There exists no social choice correspondence that satisfies Non-Emptiness, Pareto Efficiency, and $\bar{r}$ Egalitarian-Equivalence.

\footnotetext{
${ }^{11}$ We use Leontief preferences only for easy calculations. An example can be constructed with smooth and strictly monotonic preferences
} 


\section{The Efficiency-First Principle}

The results in the previous section show that we cannot always select an allocation that is both Pareto efficient and equitable. Therefore, in cases where the two criteria are conflicting with each other, we have to give priority to one of them. In this section, we adopt the efficiency criterion as the first principle, keeping the requirement of Pareto Efficiency on social choice correspondences. As for equity criteria, however, we only require their conditional versions.

By the definitions of axioms, Pareto Efficiency and P-Conditional No-Envy (or P-Conditional Egalitarian-Equivalence, P-Conditional $\bar{r}$ Egalitarian-Equivalence) together are compatible with Non-Emptiness. We examine with which choice-consistency conditions these axioms are compatible. In order to present the results, we introduce several social preference relations.

\subsection{No-Envy as the Second Criterion}

Let $R_{N} \in \mathcal{R}^{n}$ be given. We define the equity-as-no-envy superior relation, denoted $\succ_{F\left(R_{N}\right)}$, as follows: For all $x, y \in X, x \succ_{F\left(R_{N}\right)} y$ if and only if $x$ is envy-free and $y$ is not. Recall that $\succ_{P\left(R_{N}\right)}$ denotes the Pareto superior relation.

Given $R_{N} \in \mathcal{R}^{n}$, define the binary relation $\succ_{P F\left(R_{N}\right)}$ on $X$ as follows: For all $x, y \in X, x \succ_{P F\left(R_{N}\right)} y$ if and only if (i) $x \succ_{P\left(R_{N}\right)} y$ or (ii) $x \nsucc_{P\left(R_{N}\right)} y, y \nsucc_{P\left(R_{N}\right)} x$, and $x \succ_{F\left(R_{N}\right)} y$.

Under the social preference relation $\succ_{P F\left(R_{N}\right)}$, we first apply the Pareto criterion when we rank any two allocations. Then, only when the Pareto criterion does not give a strict ranking between the two, we apply the equity-as-no-envy criterion.

The next lemma clarifies the relation between the social choice correspondences satisfying Pareto Efficiency, P-Conditional No-Envy, and Contraction Consistency, and the social preference relation $\succ_{P F\left(R_{N}\right)}$.

Lemma 1. If a social choice correspondence $\varphi$ satisfies Pareto Efficiency, P-Conditional No-Envy, and Contraction Consistency, then $\varphi\left(R_{N}, S\right) \subseteq M_{\succ_{P F\left(R_{N}\right)}}(S)$ for all $\left(R_{N}, S\right) \in \mathcal{R}^{n} \times \mathcal{S}$.

Proof. Suppose that a social choice correspondence $\varphi$ satisfies Pareto Efficiency, P-Conditional No-Envy, and Contraction Consistency. Let $\left(R_{N}, S\right) \in \mathcal{R}^{n} \times \mathcal{S}$ be given. Suppose, on the contrary, that there exists $x \in S$ such that $x \in \varphi\left(R_{N}, S\right)$ but $x \notin M_{\succ_{P F\left(R_{N}\right)}}(S)$. Then, there exists $y \in S$ such that $y \succ_{P F\left(R_{N}\right)} x$. Because $x \in \varphi\left(R_{N}, S\right) \subseteq P\left(R_{N}, S\right)$ 
by Pareto Efficiency, $y \nsucc_{P\left(R_{N}\right)} x$. Hence, $y \succ_{P F\left(R_{N}\right)} x$ holds only if $x \succ_{P\left(R_{N}\right)} y$ and $y \succ_{F\left(R_{N}\right)} x$. Let $S^{\prime}=\{x, y\}$. Then, $S^{\prime} \subseteq S$ and $P\left(R_{N}, S^{\prime}\right)=S^{\prime}$. By P-Conditional No-Envy, $\varphi\left(R_{N}, S^{\prime}\right) \subseteq F\left(R_{N}, S^{\prime}\right)$. Thus, $x \notin \varphi\left(R_{N}, S^{\prime}\right)$. This means, however, that $\varphi$ violates Contraction Consistency, which is a contradiction.

It is well-known that for any binary relation $\succ$, the set $M_{\succ}(S)$ is non-empty for all $S \in \mathcal{S}$ if and only if $\succ$ is acyclic (Sen, 1970). Hence, it follows from the above lemma that there exist social choice correspondences satisfying the three axioms and Non-Emptiness only if $\succ_{P F\left(R_{N}\right)}$ is acyclic. Unfortunately, the social preference relation $\succ_{P F\left(R_{N}\right)}$ may have a cycle.

Proposition 1. [Tadenuma (2002)] There exists a preference profile $R_{N} \in \mathcal{R}^{n}$ such that $\succ_{P F\left(R_{N}\right)}$ has a cycle.

Proof. For simplicity of presentation, we consider a two-agent and twogood economy. Similar examples can be constructed for the case of any numbers of agents and goods. Let $N=\{1,2\}$ be the set of agents. Assume that agent $i \in N$ has the preference relation $R_{i}$ on $\mathbb{R}_{+}^{2}$ that is represented by the following utility function:

$$
\begin{aligned}
& u_{1}\left(x_{11}, x_{12}\right)=x_{11} x_{12} \\
& u_{2}\left(x_{21}, x_{22}\right)=2 x_{21}+x_{22}
\end{aligned}
$$

Define four allocations $x, y, z$ and $w$ by $x=((1,9),(9,1)), y=$ $((3,6),(7,4)), z=((2,8),(8,2))$, and $w=((2,7),(8,3))$. Then, $x \succ_{P F\left(R_{N}\right)} y$ since $x \nsucc_{P\left(R_{N}\right)} y, y \nsucc_{P\left(R_{N}\right)} x$, and $x \succ_{F\left(R_{N}\right)} y$. Since $y \succ_{P\left(R_{N}\right)} z$, we have $y \succ_{P F\left(R_{N}\right)} z$. Because $z \succ_{P\left(R_{N}\right)} w, w \nsucc_{P\left(R_{N}\right)} z$, and $z \succ_{F\left(R_{N}\right)} w$, we have $z \succ_{P F\left(R_{N}\right)} w$. Finally, $w \succ_{P F\left(R_{N}\right)} x$ follows from the fact that $w \succ_{P\left(R_{N}\right)} x$. Thus, the relation $\succ_{P F\left(R_{N}\right)}$ has a cycle.

From Lemma 1 and Proposition 1, the next impossibility theorem follows.

Theorem 3. There exists no social choice correspondence that satisfies Non-Emptiness, Pareto Efficiency, P-Conditional No-Envy, and Contraction Consistency.

We have argued that Path Independence is an indispensable property of social choice correspondences. However, since Path Independence implies Contraction Consistency, we have the following impossibility as a corollary of Theorem 3 . 
Corollary 1. There exists no social choice correspondence that satisfies Non-Emptiness, Pareto Efficiency, P-Conditional No-Envy, and Path Independence.

\subsection{Egalitarian-Equivalence as the Second Criterion}

Next, we adopt egalitarian-equivalence as the concept of equity instead of no-envy. The analyses will go parallel to those in the previous subsection. We define the equity-as-egalitarian-equivalence superior relation $\succ_{E\left(R_{N}\right)}$ as $x \succ_{E\left(R_{N}\right)} y$ if and only if $x$ is egalitarian-equivalent and $y$ is not. Then, define the binary relation $\succ_{P E\left(R_{N}\right)}$ on $X$ as $x \succ_{P E\left(R_{N}\right)} y$ if and only if (i) $x \succ_{P\left(R_{N}\right)} y$ or (ii) $x \succ_{P\left(R_{N}\right)} y, y \nsucc_{P\left(R_{N}\right)} x$, and $x \succ_{E\left(R_{N}\right)} y$.

Just like Lemma 1, we can show that if a social choice correspondence $\varphi$ satisfies Pareto Efficiency, P-Conditional EgalitarianEquivalence, and Contraction Consistency, then $\varphi\left(R_{N}, S\right) \subseteq$ $M_{\succ P E\left(R_{N}\right)}(S)$ for all $\left(R_{N}, S\right) \in \mathcal{R}^{n} \times \mathcal{S}$. Hence, whether the social preference relation $\succ_{P E\left(R_{N}\right)}$ is acyclic or not is a crucial question for the existence of social choice correspondence satisfying the three axioms and Non-Emptiness. However, $\succ_{P E\left(R_{N}\right)}$ may have a cycle.

Proposition 2. [Tadenuma (2005)]. There exists a preference profile $R_{N} \in \mathcal{R}^{n}$ such that $\succ_{P E\left(R_{N}\right)}$ has a cycle.

Proof. For simplicity of presentation, we consider a two-agent and twogood economy. Let $N=\{1,2\}$ be the set of agents. Assume that agent 1 's preference relation $R_{1}$ on $\mathbb{R}_{+}^{2}$ is represented by a Leontief utility function:

$$
u_{1}\left(x_{11}, x_{12}\right)=\min \left\{x_{11}, x_{12}\right\}
$$

Agent 2's preference relation $R_{2}$ is represented by the following piecewise linear utility function:

$$
\begin{aligned}
& u_{2}\left(x_{21}, x_{22}\right)=x_{21}+20 \text { if } x_{22} \geq x_{21} \text { and } x_{22} \geq 20 \\
& u_{2}\left(x_{21}, x_{22}\right)=x_{21}+x_{22} \text { if } x_{22} \geq x_{21} \text { and } x_{22} \leq 20 \\
& u_{2}\left(x_{21}, x_{22}\right)=2 x_{22} \text { if } x_{22} \leq x_{21}
\end{aligned}
$$

Define four allocations $x, y, z$ and $w$ by $x=((18,9),(10,19)), y=$ $((12,10),(16,18)), z=((23,11),(5,17))$ and $w=((17,15),(11,13))$. Then, observe the following facts: (1) $y \succ_{P\left(R_{N}\right)} x$. (2) $w \succ_{P\left(R_{N}\right)} z$. (3) $y \nsucc_{P\left(R_{N}\right)} z$ and $z \nsucc_{P\left(R_{N}\right)} y$. (4) $x \nsucc_{P\left(R_{N}\right)} w$ and $w \nsucc_{P\left(R_{N}\right)} x$.

(5) $x$ is egalitarian-equivalent with a reference bundle $(9,20)$ since 
$u_{1}\left(x_{1}\right)=9=u_{1}(9,20)$ and $u_{2}\left(x_{2}\right)=29=u_{2}(9,20) .(6) z$ is egalitarianequivalent with a reference bundle $(11,11)$. (7) $y$ is not egalitarianequivalent because for all $a_{0} \in \mathbb{R}_{+}^{2}$ such that $u_{2}\left(a_{0}\right)=u_{2}\left(y_{2}\right)=34$, $a_{0} \geq(13,13)$, and hence $u_{1}\left(a_{0}\right) \geq u_{1}(13,13)=13>10=u_{1}\left(y_{1}\right)$. (8) $w$ is not egalitarian-equivalent since for all $b_{0} \in \mathbb{R}_{+}^{2}$ such that $u_{1}\left(b_{0}\right)=u_{1}\left(w_{1}\right)=15, b_{0} \geq(15,15)$, and thus $u_{2}\left(b_{0}\right) \geq u_{2}(15,15)=$ $30>24=u_{2}\left(w_{2}\right)$.

By (1), we have $y \succ_{P E\left(R_{N}\right)} x$. It follows from (3), (6) and (7) that $z \succ_{P E\left(R_{N}\right)} y$. By $(2), w \succ_{P E\left(R_{N}\right)} z$. Finally, from (4), (5) and (8) together, we have $x \succ_{P E\left(R_{N}\right)} w$. Thus, the relation $\succ_{P E\left(R_{N}\right)}$ has a cycle.

By Proposition 2, we have the following impossibility results.

Theorem 4. There exists no social choice correspondence that satisfies Non-Emptiness, Pareto Efficiency, P-Conditional EgalitarianEquivalence, and Contraction Consistency.

Corollary 2. There exists no social choice correspondence that satisfies Non-Emptiness, Pareto Efficiency, P-Conditional EgalitarianEquivalence, and Path Independence.

\section{$5.3 \bar{r}$-Egalitarian-Equivalence as the Second Criterion}

We have reached an impossibility again with egalitarian-equivalence as the second criterion. In this subsection, we adopt a more restricted concept of equity than egalitarian-equivalence, namely $\bar{r}$-egalitarianequivalence. Let us recall that the reference bundles of $\bar{r}$-egalitarianequivalent allocations must lie in the given ray from the origin, while there is no such restriction in the definition of (general) egalitarianequivalent allocations. With $\bar{r}$-egalitarian-equivalence as the second criterion, we have a positive result as shown next.

Theorem 5. There exists a social choice correspondence that satisfies Non-Emptiness, Pareto Efficiency, P-Conditional $\bar{r}$-EgalitarianEquivalence and Path Independence.

Proof. The proof relies on the social preference relation introduced by Pazner and Schmeidler (1978). For each $R_{N} \in \mathcal{R}^{n}$, define a binary relation $\succsim_{P S\left(R_{N}\right)}$ on $\mathbb{R}_{+}^{m n}$ as follows. For all $x, y \in X, x \succsim_{P S\left(R_{N}\right)} y$ if and only if

$$
\min _{i \in N} \min \left\{\lambda_{i} \in \mathbb{R} \mid \lambda_{i} \bar{r} R_{i} x_{i}\right\} \geq \min _{i \in N} \min \left\{\lambda_{i} \in \mathbb{R} \mid \lambda_{i} \bar{r} R_{i} y_{i}\right\} .
$$


Let $\succ_{P S\left(R_{N}\right)}$ be the strict part of $\succsim_{P S\left(R_{N}\right)}$. Define the social choice function $\varphi_{P S}$ by $\varphi_{P S}\left(R_{N}, S\right)=M_{\succ_{P S\left(R_{N}\right)}}(S)$ for all $\left(R_{N}, S\right) \in \mathcal{R}^{n} \times \mathcal{S}$. It is easy to check that $\varphi_{P S}$ satisfies Non-Emptiness, Pareto Efficiency, and P-Conditional $\bar{r}$-Egalitarian-Equivalence. Since $\succsim_{P S\left(R_{N}\right)}$ is transitive and complete, and transitive rationalizability implies Path Independence, it follows that $\varphi_{P S}$ satifies Path Independence.

We argued that if explicitly defined criteria of efficiency and equity should be the only selection principles, then the stronger version of conditional efficiency and equity should be accepted. That is, if the first principle selects all or none, then the allocations satisfying the second principle should be all taken, and no discrimination between them should be introduced. Next we examine the compatibility of this stronger version with choice-consistency.

We define the equity-as- $\bar{r}$-egalitarian-equivalence superior relation $\succ_{E_{\bar{r}}\left(R_{N}\right)}$ as $x \succ_{E_{\bar{r}}\left(R_{N}\right)} y$ if and only if $x$ is $\bar{r}$-egalitarian-equivalent and $y$ is not.

Theorem 6. There exists a social choice correspondence that satisfies Non-Emptiness, Pareto Efficiency, P-Conditional $\bar{r}$-EgalitarianEquivalence Inclusion, $E_{\bar{r}}$-Conditional Pareto Inclusion, and Contraction Consistency.

Proof. For each $R_{N} \in \mathcal{R}^{n}$, define the binary relation $\succ_{P E_{\bar{r}}\left(R_{N}\right)}$ as follows. For all $x, y \in X, x \succ_{P E_{\bar{r}}\left(R_{N}\right)} y$ if and only if (i) $x \succ_{P\left(R_{N}\right)} y$ or (ii) $x \nsucc_{P\left(R_{N}\right)} y, y \nsucc_{P\left(R_{N}\right)} x$, and $x \succ_{E_{\bar{r}}\left(R_{N}\right)} y$. Define the social choice correspondence $\varphi_{P E_{\bar{r}}}$ by $\varphi_{P E_{\bar{r}}}\left(R_{N}, S\right)=M_{\succ_{P E_{\bar{r}}\left(R_{N}\right)}}(S)$ for all $\left(R_{N}, S\right) \in \mathcal{R}^{n} \times \mathcal{S}$. By Tadenuma (2005, Prop. 5), $\succ_{P E_{\bar{r}}\left(R_{N}\right)}$ is acyclic, and hence $\varphi_{P E_{\bar{r}}}$ satisfies Non-Emptiness. It is clear that $\varphi_{P E_{\bar{r}}}$ satisfies Pareto Efficiency. To check that it satisfies P-Conditional $\bar{r}$-Egalitarian-Equivalence Inclusion, let $\left(R_{N}, S\right) \in \mathcal{R}^{n} \times \mathcal{S}$ be such that $P\left(R_{N}, S\right)=S$. Clearly, $\varphi_{P E_{\bar{r}}}(S) \subseteq E_{\bar{r}}\left(R_{N}, S\right)$. Suppose that there exists $x \in E_{\bar{r}}\left(R_{N}, S\right)$ such that $x \notin \varphi_{P E_{\bar{r}}}$. Then, there is $y \in S$ with $y \succ_{P E_{\bar{r}}\left(R_{N}\right)} x$. Since $x, y \in P\left(R_{N}, S\right), y$ cannot be Pareto superior to $x$. Because $x \in E_{\bar{r}}\left(R_{N}, S\right), y$ cannot be equityas- $\bar{r}$-egalitarian-equivalence superior to $x$, either. This is a contradiction. Hence, $E_{\bar{r}}\left(R_{N}, S\right) \subseteq \varphi_{P E_{\bar{r}}}(S)$. Similarly, we can show that $\varphi_{P E_{\bar{r}}}$ satisfies $E_{\bar{r}}$-Conditional Pareto Inclusion. Finally, since $\varphi_{P E_{\bar{r}}}\left(R_{N}, \cdot\right)$ is rationalizable by the binary relation $\succ_{P E_{\bar{r}}\left(R_{N}\right)}$, it satisfies Contraction Consistency.

However, if we strengthens the requirement of choice-consistency, we have another impossibility. 
Theorem 7. There exists no social choice correspondence that satisfies Non-Emptiness, Pareto Efficiency, P-Conditional $\bar{r}$-EgalitarianEquivalence, $E_{\bar{r}}$-Conditional Pareto Inclusion, and Path Independence.

Proof. Consider the economy defined in the proof of Proposition 2. Let $\bar{r}=(9,20)$. Define three allocations $x, y$ and $w$ by $x=$ $((18,9),(10,19)), y=((12,10),(16,18))$ and $w=((17,15),(11,13))$. Then, the allocation $x$ is $\bar{r}$-egalitarian-equivalent, but the other two allocations are not. On the other hand, $y \succ_{P\left(R_{N}\right)} x$, but $y \nsucc_{P\left(R_{N}\right)} w$ and $w \nsucc_{P\left(R_{N}\right)} y$. Similarly, $x \nsucc_{P\left(R_{N}\right)} w$ and $w \nsucc_{P\left(R_{N}\right)} x$.

Suppose that there exists a social choice correspondence $\varphi$ that satisfies Pareto Efficiency, P-Conditional $\bar{r}$-Egalitarian-Equivalence, $E_{\bar{r}^{-}}$ Conditional Pareto Inclusion, Path Independence and Non-Emptiness. It follows from Pareto Efficiency, P-Conditional $\bar{r}$-EgalitarianEquivalence, and Path Independence (which implies Contraction Consistency) that $\varphi\left(R_{N}, S\right) \subseteq M_{\succ_{P E_{\bar{r}}\left(R_{N}\right)}}(S)$ for all $\left(R_{N}, S\right) \in \mathcal{R}^{n} \times \mathcal{S}$.

Let $S_{1}=\{x, y, w\}, S_{2}=\{x, y\}$ and $S_{3}=\{w\}$. Then, $M_{\succ_{P E_{\bar{r}}\left(R_{N}\right)}}\left(S_{1}\right)=\{y\}$ and $M_{\succ_{P E_{\bar{r}}\left(R_{N}\right)}}\left(S_{2}\right)=\{y\}$. By the above relation and Non-Emptiness of $\varphi$, we have

$$
\varphi\left(R_{N}, S_{1}\right)=\{y\} \text { and } \varphi\left(R_{N}, S_{2}\right)=\{y\}
$$

By Non-Emptiness, $\varphi\left(R_{N}, S_{3}\right)=\{w\}$. Hence,

$$
\varphi\left(R_{N}, \varphi\left(R_{N}, S_{2}\right) \cup \varphi\left(R_{N}, S_{3}\right)\right)=\varphi\left(R_{N},\{y, w\}\right)
$$

Observe that $E_{\bar{r}}\left(R_{N},\{y, w\}\right)=\emptyset$ and $P\left(R_{N},\{y, w\}\right)=\{y, w\}$. Since $\varphi$ satisfies $E_{\bar{r}}$-Conditional Pareto Inclusion, we have

$$
\varphi\left(R_{N},\{y, w\}\right)=\{y, w\}
$$

It follows from (1), (2) and (3) that $\varphi\left(R_{N}, \varphi\left(R_{N}, S_{2}\right) \cup \varphi\left(R_{N}, S_{3}\right)\right) \neq$ $\varphi\left(R_{N}, S_{1}\right)$. This means that $\varphi$ violates Path Independence, which is a contradiction.

\section{The Equity-First Principle}

In this section, we reverse the order of application of the efficiency and equity criteria. That is, we first select from equitable allocations, and if the equity criterion is not effective as a guide for selection either because all the available allocations are equitable or because no allocation is equitable, then we choose from efficient allocations. In the following, we consider the equity-as-no-envy criterion. However, 
essentially the same results hold true with egalitarian-equivalence or $\bar{r}$-egalitarian-equivalence.

To identify the social choice correspondences satisfying No-Envy, FConditional Pareto Efficiency and Contraction Consistency, it is useful to introduce a social preference relation. Given $R_{N} \in \mathcal{R}^{n}$, define the binary relation $\succ_{F P\left(R_{N}\right)}$ on $X$ as follows: For all $x, y \in X, x \succ_{F P\left(R_{N}\right)} y$ if and only if (i) $x \succ_{F\left(R_{N}\right)} y$ for $R_{N}$, or (ii) $x \nsucc_{F\left(R_{N}\right)} y, y \nsucc_{F\left(R_{N}\right)} x$, and $x \succ_{P\left(R_{N}\right)} y$. Under this social preference relation, we first apply the equity-as-no-envy criterion to rank any two allocations, and when the two allocations are not strictly ranked by the criterion because both are envy-free or neither is, we invoke the efficiency criterion.

The next lemma is the counterpart of Lemma 1.

Lemma 2. If a social choice correspondence $\varphi$ satisfies No-Envy, F-Conditional Pareto Efficiency, and Contraction Consistency, then $\varphi\left(R_{N}, S\right) \subseteq M_{\succ F\left(R_{N}\right)}(S)$ for all $\left(R_{N}, S\right) \in \mathcal{R}^{n} \times \mathcal{S}$.

Proof. Suppose that a social choice correspondence $\varphi$ satisfies NoEnvy, F-Conditional Pareto Efficiency, and Contraction Consistency. Let $\left(R_{N}, S\right) \in \mathcal{R}^{n} \times \mathcal{S}$ be given. Suppose, on the contrary, that there exists $x \in S$ such that $x \in \varphi\left(R_{N}, S\right)$ but $x \notin M_{\succ_{F P\left(R_{N}\right)}}(S)$. Then, there exists $y \in S$ such that $y \succ_{F P\left(R_{N}\right)} x$. We distinguish two cases.

Case 1: $F\left(R_{N}, S\right) \neq \emptyset$.

Because $x \in \varphi\left(R_{N}, S\right) \subseteq F\left(R_{N}, S\right)$ by No-Envy, $y \succ_{F P\left(R_{N}\right)} x$ holds only if both $x$ and $y$ are envy-free, and $y$ is Pareto superior to $x$. Let $S^{\prime}=\{x, y\}$. Then, $S^{\prime} \subseteq S$, and $F\left(R_{N}, S^{\prime}\right)=S^{\prime}$. By F-Conditional Pareto Efficiency, $\varphi\left(R_{N}, S^{\prime}\right) \subseteq P\left(R_{N}, S^{\prime}\right)=\{y\}$. Thus, $x \notin \varphi\left(R_{N}, S^{\prime}\right)$. This means that $\varphi$ violates Contraction Consistency, which is a contradiction.

Case 2: $F\left(R_{N}, S\right)=\emptyset$.

Then, $y \succ_{F P\left(R_{N}\right)} x$ holds only if $y$ is Pareto superior to $x$. Let $S^{\prime}=$ $\{x, y\}$. Then, $S^{\prime} \subseteq S$, and $F\left(R_{N}, S^{\prime}\right)=\emptyset$. By F-Conditional Pareto Efficiency, $\varphi\left(R_{N}, S^{\prime}\right) \subseteq P\left(R_{N}, S^{\prime}\right)=\{y\}$. The rest of the argument is the same as Case 1 .

The next result should be contrasted with Proposition 1.

Proposition 3. For all $R_{N} \in \mathcal{R}^{n}, \succ_{F P\left(R_{N}\right)}$ is transitive.

Proof. Let $R_{N} \in \mathcal{R}^{n}$ be given. To lighten notation, we simply write $\succ_{F P}, \succ_{P}$, and $F$ for $\succ_{F P\left(R_{N}\right)}, \succ_{P\left(R_{N}\right)}$, and $F\left(R_{N}, X\right)$, respectively. Assume that $x \succ_{F P} y$ and $y \succ_{F P} z$. By $x \succ_{F P} y,(1) x \in F$ and $y \notin F$ or $(2)[[x \in F$ and $y \in F]$ or $[x \notin F$ and $y \notin F]]$ and $x \succ_{P} y$. By $y \succ_{F P}$ 
$z,(3) y \in F$ and $z \notin F$ or (4) $[[y \in F$ and $z \in F]$ or $[y \notin F$ and $z \notin F]]$ and $y \succ_{P} z$. (1) and (3) are incompatible. If (1) and (4) hold, then we must have $x \in F$ and $z \notin F$. Hence, $x \succ_{F P} z$. Similarly, if (2) and (3) hold, then $x \in F$ but $z \notin F$, and we have $x \succ_{F P} z$. If (2) and (4) hold, then either $x, y, z \in F$ and $x \succ_{P} y \succ_{P} z$ or $x, y, z \notin F$ and $x \succ_{P} y \succ_{P} z$. Since the relation $\succ_{P}$ is transitive, we have $x \succ_{F P} z$.

Define the social choice correspondence $\varphi_{F P}$ by

$$
\varphi_{F P}\left(R_{N}, S\right)=M_{\succ_{F P\left(R_{N}\right)}}(S) \text { for all }\left(R_{N}, S\right) \in \mathcal{R}^{n} \times \mathcal{S} .
$$

This correspondence takes the following values:

$$
\begin{aligned}
& \varphi_{F P}\left(R_{N}, S\right)=P\left(R_{N}, F\left(R_{N}, S\right)\right) \text { if } F\left(R_{N}, S\right) \neq \emptyset \\
& \varphi_{F P}\left(R_{N}, S\right)=P\left(R_{N}, S\right) \text { if } F\left(R_{N}, S\right)=\emptyset
\end{aligned}
$$

Note that for all $\left(R_{N}, S\right) \in \mathcal{R}^{n} \times \mathcal{S}, P\left(R_{N}, F\left(R_{N}, S\right)\right) \supseteq$ $P\left(R_{N}, S\right) \cap F\left(R_{N}, S\right)$ and there exists $\left(R_{N}, S\right) \in \mathcal{R}^{n} \times \mathcal{S}$ such that $P\left(R_{N}, F\left(R_{N}, S\right)\right) \neq P\left(R_{N}, S\right) \cap F\left(R_{N}, S\right)$.

We next show that the social choice correspondence $\varphi_{F P}$ is characterized by No-Envy, the stronger versions of conditional Pareto Efficiency and No-Envy, and Path Independence.

Theorem 8. A social choice correspondence $\varphi$ satisfies NonEmptiness, No-Envy, P-Conditional No-Envy Inclusion, F-Conditional Pareto Inclusion, and Path Independence if and only if $\varphi=\varphi_{F P}$.

Proof. First, we show that $\varphi_{F P}$ satisfies the five axioms. It is easy to check that $\varphi_{F P}$ satisfies No-Envy, F-Conditional Pareto Inclusion and P-Conditional No-Envy Inclusion. By Proposition $3, \varphi_{F P}$ satisfies Quasi-Transitive Rationalizability, which implies Path Independence. Since any quasi-transitive binary relation has maximal elements in any finite set, Non-Emptiness follows.

Next we show that $\varphi_{F P}$ is the unique social choice correspondence that satifies the five axioms. Suppose, on the contrary, that there is a social choice correspondence $\varphi$ with $\varphi \neq \varphi_{F P}$ that satisfies the five axioms. Then, there is $\left(R_{N}, S\right) \in \mathcal{R}^{n} \times \mathcal{S}$ such that

$$
\varphi\left(R_{N}, S\right) \neq \varphi_{F P}\left(R_{N}, S\right)
$$

If $F\left(R_{N}, S\right)=\emptyset$, then by F-Conditional Pareto Inclusion, $\varphi\left(R_{N}, S\right)=$ $P\left(R_{N}, S\right)$. On the other hand, it can be checked that $\varphi_{F P}\left(R_{N}, S\right)=$ $M_{\succ_{F P\left(R_{N}\right)}}(S)=P\left(R_{N}, S\right)$. Hence, we have $\varphi\left(R_{N}, S\right)=\varphi_{F P}\left(R_{N}, S\right)$, a 
contradiction. Thus, $F\left(R_{N}, S\right) \neq \emptyset$. Since F-Conditional Pareto Inclusion implies F-Conditional Pareto Efficiency, and Path Independence implies Contraction Consistency, it follows from Lemma 2 that

$$
\varphi\left(R_{N}, S\right) \subseteq M_{\succ_{F P\left(R_{N}\right)}}(S)=\varphi_{F P}\left(R_{N}, S\right)
$$

Because $F\left(R_{N}, S\right) \neq \emptyset$, we have

$$
M_{\succ_{F P\left(R_{N}\right)}}(S)=P\left(R_{N}, F\left(R_{N}, S\right)\right)
$$

It follows from (1), (2) and (3) that there exists $x^{*} \in S$ such that $x^{*} \in$ $P\left(R_{N}, F\left(R_{N}, S\right)\right)$ but $x^{*} \notin \varphi\left(R_{N}, S\right)$. Define $S^{\prime}:=\left\{x^{*}\right\} \cup\{y \in S \mid y \in$ $F\left(R_{N}, S\right)$ and $\left.x^{*} \succ_{P\left(R_{N}\right)} y\right\} \cup\left[S \backslash F\left(R_{N}, S\right)\right]$. By Lemma 2, $\varphi\left(R_{N}, S^{\prime}\right) \subseteq$ $M_{\succ F P\left(R_{N}\right)}\left(S^{\prime}\right)=\left\{x^{*}\right\}$. By Non-Emptiness, we have $\varphi\left(R_{N}, S^{\prime}\right)=\left\{x^{*}\right\}$. Define $S^{\prime \prime}:=S \backslash S^{\prime}$. Again from Lemma 2, it follows that

$$
\varphi\left(R_{N}, S^{\prime \prime}\right) \subseteq M_{\succ_{F P\left(R_{N}\right)}}\left(S^{\prime \prime}\right)=P\left(R_{N}, F\left(R_{N}, S^{\prime \prime}\right)\right)
$$

Claim: $P\left(R_{N}, F\left(R_{N}, S^{\prime \prime}\right)\right) \subset P\left(R_{N}, F\left(R_{N}, S\right)\right)$.

Let $z \in P\left(R_{N}, F\left(R_{N}, S^{\prime \prime}\right)\right)$. Then, $z \in F\left(R_{N}, S^{\prime \prime}\right) \subset F\left(R_{N}, S\right)$. Suppose that $z \notin P\left(R_{N}, F\left(R_{N}, S\right)\right)$. Then, there exists $w \in$ $P\left(R_{N}, F\left(R_{N}, S\right)\right)$ such that $w \succ_{P\left(R_{N}\right)} z$. If $w=x^{*}$, then $z \in S^{\prime}$ and hence $z \notin S^{\prime \prime}$, which is a contradiction. Thus, $w \neq x^{*}$. But then, $w \in S^{\prime \prime}$ and so $z \notin P\left(R_{N}, F\left(R_{N}, S^{\prime \prime}\right)\right)$, which contradicts $z \in P\left(R_{N}, F\left(R_{N}, S^{\prime \prime}\right)\right)$. Therefore, we must have $z \in P\left(R_{N}, F\left(R_{N}, S\right)\right)$. Thus, the claim has been proved.

It follows from (4) and the above claim that $\varphi\left(R_{N}, S^{\prime \prime}\right) \subseteq$ $P\left(R_{N}, F\left(R_{N}, S\right)\right)$. Hence, $\varphi\left(R_{N}, S^{\prime}\right) \cup \varphi\left(R_{N}, S^{\prime \prime}\right)=\left\{x^{*}\right\} \cup \varphi\left(R_{N}, S^{\prime \prime}\right) \subseteq$ $P\left(R_{N}, F\left(R_{N}, S\right)\right)$. Therefore, $P\left(R_{N}, \varphi\left(R_{N}, S^{\prime}\right) \cup \varphi\left(R_{N}, S^{\prime \prime}\right)\right)=$ $\varphi\left(R_{N}, S^{\prime}\right) \cup \varphi\left(R_{N}, S^{\prime \prime}\right)$. Then, by P-Conditional No-Envy Inclusion, we conclude that $\varphi\left(R_{N}, \varphi\left(R_{N}, S^{\prime}\right) \cup \varphi\left(R_{N}, S^{\prime \prime}\right)\right)=F\left(R_{N}, \varphi\left(R_{N}, S^{\prime}\right) \cup\right.$ $\left.\varphi\left(R_{N}, S^{\prime \prime}\right)\right)=\varphi\left(R_{N}, S^{\prime}\right) \cup \varphi\left(R_{N}, S^{\prime \prime}\right)=\left\{x^{*}\right\} \cup \varphi\left(R_{N}, S^{\prime \prime}\right)$. But since $x^{*} \notin \varphi\left(R_{N}, S\right)=\varphi\left(R_{N}, S^{\prime} \cup S^{\prime \prime}\right)$ and $\varphi$ satisfies Path Independence, we must have $x^{*} \notin \varphi\left(R_{N}, \varphi\left(R_{N}, S^{\prime}\right) \cup \varphi\left(R_{N}, S^{\prime \prime}\right)\right)$. This is a contradiction.

Therefore, there is no social choice correspondence $\varphi$ with $\varphi \neq \varphi_{F P}$ that satisfies the five axioms together in the statement of the theorem.

\section{Minimal-Envy and Choice-Consistency}

In the previous sections, our equity-as-no-envy criterion made only "allor-nothing" selection: an allocation is selected if there is no envy at 
all, whereas it is not selected if there is at least one instance of envy. However, among allocations with envy, the instances of envy may differ greatly. In such cases, it should be desirable to select allocations with "minimal" instances of envy.

In this section, we introduce a measure of envy at allocations, which is due to Suzumura (1996). Based on the measure, we define the notion of minimal-envy. Then, as in the foregoing section, we examine the choice-consistency properties of social choice correspondences satisfying the efficiency first or the equity first principle with minimal-envy as the concept of equity.

For each $R_{N} \in \mathcal{R}^{n}$, and each $x \in X$, define the set $H\left(R_{N}, x\right) \subset$ $N \times N$ by

$$
H\left(R_{N}, x\right)=\left\{(i, j) \in N \times N \mid x_{j} P_{i} x_{i}\right\}
$$

The set $H\left(R_{N}, x\right)$ is the set of all instances of envy at $x$. Following Suzumura (1996), we define the binary relation. $\succ_{F_{\min }\left(R_{N}\right)}$ as follows: for all $x, y \in X, x \succ_{F_{\min }\left(R_{N}\right)} y$ if and only if $H\left(R_{N}, x\right) \subsetneq H\left(R_{N}, y\right)$.

In a similar way to defining Pareto efficiency, we can define the notion of minimal-envy. Given $\left(R_{N}, S\right) \in \mathcal{R}^{n} \times \mathcal{S}$, an allocation $x \in S$ is envy-minimal in $S$ for $R_{N}$ if there is no allocation $y \in S$ such that $H\left(R_{N}, y\right) \subsetneq H\left(R_{N}, x\right)$. Let $F_{\min }\left(R_{N}, S\right)$ be the set of envy-minimal allocations in $S$ for $R_{N}$. By simply replacing $F\left(R_{N}, S\right)$ with $F_{\min }\left(R_{N}, S\right)$ in the definitions of axioms in Section 3, we can define axioms, MinimalEnvy, P-Conditional Minimal-Envy, and $F_{\min }$-Conditional Pareto Efficiency.

Reexamining the proofs of Lemma 1, Proposition 1 and Theorem 3 , we find that there is no social choice correspondence that satisfies Non-Emptiness, Pareto Efficiency, P-Conditional Minimal-Envy and Contraction Consistency. Moreover, with this concept of equity, even the equity-first principle may contradict the minimum requirement of choice-consistency, which we now turn to.

For each $R_{N} \in \mathcal{R}^{n}$, define the binary relation $\succ_{F_{\min } P\left(R_{N}\right)}$ as follows: For all $x, y \in X, x \succ_{F_{\min } P\left(R_{N}\right)} y$ if and only if (i) $x \succ_{F_{\min }\left(R_{N}\right)} y$, or (ii) $x \nsucc_{F_{\min }\left(R_{N}\right)} y, y \nsucc_{F_{\min }\left(R_{N}\right)} x$, and $x \succ_{P\left(R_{N}\right)} y$. Then, as in Lemma 2, we can show that if a social choice correspondence $\varphi$ satisfies Non-Emptiness, Minimal-Envy, $F_{\min }$-Conditional Pareto Efficiency and Contraction Consistency, then $\varphi\left(R_{N}, S\right) \subseteq M_{\succ_{F_{\min } P\left(R_{N}\right)}}(S)$ for all $\left(R_{N}, S\right) \in \mathcal{R}^{n} \times \mathcal{S}$.

Proposition 4. There exist a preference profile $R_{N} \in \mathcal{R}^{n}$ such that $\succ_{F_{\min } P\left(R_{N}\right)}$ has a cycle. 
Proof. Consider an economy with four agents and two goods. Let $N=$ $\{1,2,3,4\}$ be the set of agents. Assume that the agents' preference relations are represented by the following utility functions:

$$
\begin{aligned}
& u_{1}\left(x_{11}, x_{12}\right)=x_{11} x_{12} \\
& u_{3}\left(x_{31}, x_{32}\right)=x_{31}+x_{32}
\end{aligned}
$$

and

$$
u_{2}=u_{1} \text { and } u_{4}=u_{3} .
$$

Cosider the following three allocations, $x, y$ and $z$ :

$$
\begin{aligned}
\left(x_{1}, x_{2}, x_{3}, x_{4}\right) & =((6,0),(0,6),(3,3),(3,3)) \\
\left(y_{1}, y_{2}, y_{3}, y_{4}\right) & =((0.5,1),(0.5,6),(8.5,0),(2.5,5)) \\
\left(z_{1}, z_{2}, z_{3}, z_{4}\right) & =((1,1),(2,2),(5,4),(4,5))
\end{aligned}
$$

Observe that

$$
z \succ_{P\left(R_{N}\right)} y \succ_{P\left(R_{N}\right)} x
$$

and

$$
\begin{aligned}
& H\left(R_{N}, x\right)=\{(1,3),(1,4),(2,3),(2,4)\} \\
& H\left(R_{N}, y\right)=\{(1,4),(2,4),(1,2),(4,3)\} \\
& H\left(R_{N}, z\right)=\{(1,3),(1,4),(2,3),(2,4),(1,2)\} .
\end{aligned}
$$

Since $H\left(R_{N}, x\right) \nsubseteq H\left(R_{N}, y\right), H\left(R_{N}, y\right) \nsubseteq H\left(R_{N}, x\right)$, and $y \succ_{P\left(R_{N}\right)} x$, we have $y \succ_{F_{\min } P\left(R_{N}\right)} x$. Similarly, $z \succ_{F_{\min } P\left(R_{N}\right)} y$ holds true. However, it follows from $H\left(R_{N}, x\right) \subsetneq H\left(R_{N}, z\right)$ that $x \succ_{F_{\min } P\left(R_{N}\right)} z$. Thus, there is a cycle for $\succ_{F_{\min }} P\left(R_{N}\right)$.

In a framework of abstract social choice, Suzumura (2004) defines the binary relation $\succsim_{F_{S} P\left(R_{N}\right)}$ as follows: For all $x, y \in X, x \succsim_{F_{S} P\left(R_{N}\right)} y$ if and only if (i) $H\left(R_{N}, x\right) \subseteq H\left(R_{N}, y\right)$ or (ii) $H\left(R_{N}, x\right) \nsubseteq H\left(R_{N}, y\right)$, $H\left(R_{N}, y\right) \nsubseteq H\left(R_{N}, x\right)$, and $x \succsim_{P} y$. Let $\succ_{F_{S} P\left(R_{N}\right)}$ be the strict part of $\succsim_{F_{S} P\left(R_{N}\right)}$. Suzumura (2004) presented an example such that $\succsim_{F_{S} P\left(R_{N}\right)}$ is acyclic but is not consistent. Let us recollect that a binary relation $\succsim$ on $X$ is consistent if for any integer $k \geq 3$, there exists no finite set $\left\{x^{1}, x^{2}, \ldots, x^{k}\right\} \subseteq X$ such that $x^{1} \succ x^{2}, x^{2} \succsim x^{3}, \ldots, x^{k-1} \succsim x^{k}$, and $x^{k} \succsim x^{1}$.

Notice that there is an important difference between the definitions of $\succ_{F_{\min } P\left(R_{N}\right)}$ and $\succ_{F_{S} P\left(R_{N}\right)}$. Just as all other lexicographic compositions of two criteria studied in this paper and Tadenuma (2002, 2005), 
the relation $\succ_{F_{\min } P\left(R_{N}\right)}$ invokes the second criterion (the Pareto superior relation in this case) when the first criterion judges the two alternatives to be indifferent or non-comparable, and if there is a strict ranking between the two by the second criterion, it is adopted. In contrast, the relation $\succ_{F_{S} P\left(R_{N}\right)}$ does not make any strict ranking when the two alternatives are indifferent for the first criterion. For example, if $H\left(R_{N}, x\right)=H\left(R_{N}, y\right)$ and $x \succ_{P\left(R_{N}\right)} y$, then $x \succ_{F_{\min } P\left(R_{N}\right)} y$ but $x \nsucc_{F_{S} P\left(R_{N}\right)} y$.

A motivation for our lexicographic composition of two binary relations is that we should apply the second criterion to evaluate desirability of two allocations whenever they are non-comparable or equally good by the first criterion, so that we could have a more fine-grained social ranking of allocations. If the set of pairs of envious and envied agents is exactly the same for the two allocations, and one of them is Pareto superior to the other, why do not we choose the former? Similarly, if the two allocations give the same utility for every agent, and one of them is more equitable than the other, then we should select the more equitable.

In spite of this difference in the definitions, the proof of Proposition 4 actually shows that the relation $\succ_{F_{S} P\left(R_{N}\right)}$ also has a cycle for the preference profile because the proof does not depend on the case where the set of instances of envy is exactly the same for the two allocations. Thus, Proposition 4 strengthens Suzumura's result in two respects. First, we show that the relation $\succsim_{F_{S} P\left(R_{N}\right)}$ is not even acyclic. Note that acyclicity is a weaker condition than consistency. Second, we establish the result on the domain of classical exchange economies with no free disposal of goods and continuous, convex and strictly monotonic preferences. Even on this much restricted domain, we cannot avoid a cycle of the strict part of $\succsim_{F_{S} P\left(R_{N}\right)}$.

From Proposition 4, we obtain another impossibility theorem. The proof is analogous to those for the previous results, and it is omitted.

Theorem 9. There exists no social choice correspondence that satisfies Non-Emptiness, Minimal-Envy, $F_{\min }$-Conditional Pareto Efficiency and Contraction Consistency.

Feldman and Kirman (1974) introduced a different measure of envy at allocations, which simply counts the number of instances of envy. Based on the measure, we may define the notion of the least envy.

For each $\left(R_{N}, S\right) \in \mathcal{R}^{n} \times \mathcal{S}$, we say that an allocation $x \in S$ has the least envy in $S$ for $R_{N}$ if there is no allocation $y \in S$ such that 
$\# H\left(R_{N}, y\right)<\# H\left(R_{N}, x\right) .{ }^{12}$ Let $F_{\text {least }}\left(R_{N}, S\right)$ be the set of allocations that have the least envy in $S$. Then, we can similarly define axioms, Least-Envy, P-Conditional Least-Envy, and $F_{\text {least }}$-Conditional Pareto Efficiency.

As in Theorems 3 and 8, we can show that (i) there exists no social choice correspondence that satisfies Non-Emptiness, Pareto Efficiency, P-Conditional Least-Envy and Contraction Consistency; and (ii) there exists a social choice correspondence that satisfies NonEmptiness, Least-Envy, $F_{\text {least }}$-Conditional Pareto Efficiency and Path Independence. The proofs are similar, and we omit them.

\section{Concluding Remarks}

In his two seminal papers, Suzumura (1981a, b) considered a class of abstract social choice problems, and examined possibility of constructing social choice correspondences satisfying the following conditions on efficiency and equity.

Fairness Extension: For all $\left(R_{N}, S\right) \in \mathcal{R}^{n} \times \mathcal{S}, \varphi\left(R_{N}, S\right)=$ $P\left(R_{N}, S\right) \cap F\left(R_{N}, S\right)$ if $P\left(R_{N}, S\right) \cap F\left(R_{N}, S\right) \neq \emptyset$.

This axiom requires that if there are Pareto efficient and equitable allocations, then they should be all selected.

Fairness Inclusion: For all $\left(R_{N}, S\right) \in \mathcal{R}^{n} \times \mathcal{S}$, if $P\left(R_{N}, S\right) \cap$ $F\left(R_{N}, S\right)=\emptyset, y \in \varphi\left(R_{N}, S\right)$, and $x \in S$ is Pareto superior to $y$ or equity-as-no-envy superior to $y$ for $R_{N}$, then $x \in \varphi\left(R_{N}, S\right)$.

This means that if some allocation is selected, and there are allocations that are superior to the former either in the Pareto principle or in the equity criterion, then the latter allocations should also be selected.

A basic difference of these axioms from ours is that they treat the efficiency criterion and the equity criterion with equal weight, whereas our axioms give priority to one of the two criteria. Indeed, there is no logical relation between Fairness Extension or Fairness Inclusion and any one or any combination of our axioms concerning efficiency and equity. Moreover, combined with the requirement of Non-Emptiness, Fairness Inclusion is incompatible with either of our axioms Pareto Efficiency and No-Envy. To see this, let us reconsider the case of fundamental conflict between the Pareto criterion and the equity-as-no-envy criterion as in Section 4. In Example 1, the allocation $y$ is Pareto superior to the allocation $x$, whereas $x$ is equity-as-no-envy-superior to $y$.

${ }^{12}$ For any $\operatorname{set} A, \# A$ denotes the cardinality of $A$. 
Then, if a social choice correspondence $\varphi$ satisfies Non-Emptiness and Fairness Inclusion, then $\varphi(\{x, y\})=\{x, y\}$. That is, any correspondence satisfying this axiom avoids selection in face of the fundamental conflict. To the contrary, the correspondences satisfying our efficiency-first or equity-first axioms do make a selection in the case of the fundamental conflict, depending upon which criterion should be placed first.

Another difference between Suzumura (1981a, b) and this paper lies in the domain of social choice problems. While he considers a class of abstract social choice problems with no restrictions on individual preferences except rationality, we study the class of canonical economic problems of distributing infinitely divisible goods among $n$ agents with preferences that satisfy all standard assumptions in economics. In general, with more restrictions on the domain of problems, the more cases for compatibility among required conditions may arise. ${ }^{13}$ To put it in other words, impossibility results obtained in this paper straightforwardly extend to the unrestricted domain.

This paper started with the simple question: "Which criterion should we take first to select socially desirable allocations, the efficiency criterion or the equity criterion?" We have represented two alternative principles in the form of axioms, and examined choice-consistency of the social choice correspondences satisfying these axioms. Our results show that the existence of path independent or contraction consistent social choice correspondences depends not only on which philosophical position we take but also on what is the precise notion of equity.

There are many cases in the real life in which we must consider multiple criteria in individual or social decision-making problems. To explore general conditions for lexicographic compositions of two criteria to satisfy various degrees of choice-consistency may be an interesting topic in future researches.

\section{Acknowledgments}

This paper is dedicated to Kotaro Suzumura with my deep gratitude for his numerous comments, suggestions, and advice on my researches. I would also like to thank a referee on this paper for many useful comments. Financial support from the Japan Society for the Promotion of Science through the grant for the 21st Century Center of Excellence

${ }^{13}$ For instance, Arrow's impossibility theorem holds on the unrestricted domain, but on the restricted domain where an odd number of agents have single-peaked preferences over $\mathbb{R}$, the majority voting rule generates a complete and transitive social preference relation. 
Program on the Normative Evaluation and Social Choice of Contemporary Economic Systems and the Grant-in-Aid for Scientific Research (B) No. 18330036 is gratefully acknowledged.

\section{References}

Arrow, K. J. (1963). Social Choice and Individual Values, 2nd ed. Wiley, New York, 1 st ed. 1951.

Chernoff, H. (1954). Rational selection of decision functions. Econometrica, 22, 442-443.

Feldman, A. M. and Kirman, A. (1974). Fairness and envy. American Economic Review, 64, 996-1005.

Foley, D. (1967). Resource allocation and the public sector. Yale Economic Essays, 7, 45-98.

Kolm, S.-C. (1972). Justice et Equité. Centre National de la Recherche Scientifique, Paris.

Pazner, E. A. and Schmeidler, D. (1978). Egalitarian equivalent allocations: A new concept of economic equity. Quarterly Journal of Economics, 92, 671-687.

Plott, C. R. (1973). Path independence, rationality, and social choice. Econometrica, 41, 1075-1091.

Sen, A. K. (1970). Collective Choice and Social Welfare. Holden-Day, San Francisco.

Suzumura, K. (1981a). On Pareto-efficiency and the no-envy concept of equity. Journal of Economic Theory, 25, 367-379.

Suzumura, K. (1981b). On the possibility of 'fair' collective choice rule. International Economic Review, 22, 351-364.

Suzumura, K. (1983). Rational Choice, Collective Decisions, and Social Welfare. Cambridge University Press, Cambridge, U.K.

Suzumura, K. (1996). Interpersonal comparisons of the extended sympathy type and the possibility of social choice. In K. J. Arrow, A. K. Sen, and K. Suzumura, editors, Social Choice Re-Examined, Volume 2, pages 202-229. Macmillan, London.

Suzumura, K. (2004). An extension of Arrow's lemma with economic applications. COE/RES Discussion Paper Series No. 79, Hitotsubashi University.

Tadenuma, K. (1998). Efficiency first or equity first? Two principles and rationality of social choice. Economics Discussion Paper Series No. 1998-01, Hitotsubashi University.

Tadenuma, K. (2002). Efficiency first or equity first? Two principles and rationality of social choice. Journal of Economic Theory, 104, 462-472.

Tadenuma, K. (2005). Egalitarian-equivalence and the Pareto principle for social preferences. Social Choice and Welfare, 24, 455-473.

Thomson, W. (1996). Concepts of implementation. Japanese Economic Review, 47, $133-143$ 